\title{
Influence of facial threading on various physiological parameters of the skin: non-randomized trial involving adult women in Taiwan*
}

\author{
Li-Ying Lin ${ }^{1,2}$, Shang-Chia Chiou ${ }^{2}$
}

DOI: http:/ / dx.doi.org/10.1590/abd1806-4841.20186865

\begin{abstract}
BACKGROUND: Facial threading involves the removal of hairs to restore facial skin smoothness. However, its effectiveness has not been rigorously evaluated.

Овлестіvе: To evaluate effects of facial threading on skin roughness, hydration, melanin index, and vellus hair on the face, complemented by a subjective evaluation of the tactile feel of the skin and improvement in skin color.

METHOD: Participants who had not used exfoliators for two weeks before the experiment were included. Each participant underwent one session of facial threading every 21 days, for a total of 3 sessions. A three-dimensional skin roughness instrument and a multifunctional skin testing system were used to evaluate changes in roughness, hydration, and pigmentation on the forehead, cheeks, and corners of the mouth. A photomicrographic camera was used to record changes in vellus hair. Subjective reports of skin smoothness and color were recorded.

RESULT: Eighteen participants completed the study. Facial threading produced a significant decrease in skin roughness on the forehead $(22.42 \%, p=.013)$, right cheek $(77 \%, p=.02)$, and left corner of the mouth $(33.02 \%, p=.001)$. Subjective improvement in tactile feel of the skin and coloring were reported.

StUdy Limitations: The study did not include randomization, with further limitations of a small sample size and a single site. CONCLUSION: Facial threading reduced skin roughness by $26.74 \%$ after three threading sessions, with improved subjective assessment of tactile feel and coloring. Future research should include a comparison with other cosmetic products with similar beautifying effects or a control group.
\end{abstract}

Keywords: Beauty; Face; Hair removal; Skin care

\section{INTRODUCTION}

Facial threading is a temporary hair removal technique that is also called "Bande Abru" (Southeast Asia), "Khite" (Middle East), "Fatlah" (Egypt), or "Wanmian" (China). ${ }^{1}$ The technique involves the use of two intertwined cotton threads to remove vellus hairs and to modify the shape of the eyebrows.

According to ancient records, this beauty technique was first used in China 300 years ago. Moreover, during the Japanese
Colonial Period, records show that "hair removal" was regarded as an occupation. ${ }^{2}$ Traditionally, young women underwent their first facial threading on the eve of their marriage, symbolizing the transition to adulthood. Married women, generally, underwent facial threading on the day prior to a holiday. ${ }^{3}$ India also had a similar cultural tradition. ${ }^{4}$ Because of cultural factors, women in Taiwan believe that facial threading can remove extra hairs and make their

\footnotetext{
Received 28 December 2016.

Accepted 13 April 2017.

* Work conducted at the Chung Hwa University of Medical Technology, Tainan City, Taiwan.

Financial support: None.

Conflict of interest: None.

Department of Cosmetic Science, Chung Hwa University of Medical Technology, Tainan City, Taiwan.

Graduate School of Design, National Yunlin University of Science and Technology, Yunlin County, Taiwan.

MAILING ADDRESS:

Li-Ying Lin

E-mail: clme09@hotmail.com

C2018 by Anais Brasileiros de Dermatologia
}

(cc) BY-NC 
skin fairer and smoother. However, the effects of hair removal on improving skin roughness, hydration, and whiteness remain unproven. With cultural migration and the effects of mass media, this popular traditional Asian beauty technique is gradually becoming more common in the United States and Europe. Its increase in popularity is related to its being efficient and inexpensive, especially for removing extra hairs around the eyebrows. ${ }^{5}$ However, complications of facial threading, including irritant dermatitis, folliculitis, koebnerization, verrucae, and impetigo, have also been reported. ${ }^{6-10}$

Although facial threading carries some potential risk, many women are still interested in this natural, traditional beauty technique. Thus, an empirical evaluation of the effects of facial threading is imperative. As well, since many dermatologists have issued warnings regarding complications associated with facial threading, a determination of the validity of those reservations is also necessary. People often evaluate skin health based on its surface roughness, moisture retention, and color changes, because these factors are closely linked to wrinkle formation and female standards of aesthetics. ${ }^{11,12}$ Many cosmetic manufacturers claim that their products affect biophysical parameters, such as slowing skin aging, based on the capacity of these products to reduce roughness, increase moisture retention, and improve color. ${ }^{13-15}$

Our aim was to evaluate effects of facial threading on skin roughness, hydration, melanin index, and vellus hair on the face, complemented by a subjective evaluation of the tactile feel of the skin and improvement in skin color. To our knowledge, this is the first study to have evaluated the effects of facial threading. We propose that actual scientific evidence may allow dermatologists to educate their patients and possibly recommend facial threading as a therapeutic option to improve facial skin. Moreover, these data may provide accurate information regarding facial threading to beauty school instructors, cosmetologists, hairdressers, and the general public

\section{METHODS}

Each of the 18 female participants underwent one session of facial threading every 21 days over a period of 42 days, for a total of three sessions. A three-dimensional (3D) skin roughness instrument and a multifunctional skin analyzer were used to evaluate the physiological parameters of the skin (roughness, hydration, and melanin index), before and after facial threading. Additionally, a questionnaire-based survey was conducted before the first threading and after each subsequent threading session to obtain subjective data regarding the tactile feel of the skin and its color.

\section{Study participants}

A public presentation was organized on a university campus in Southern Taiwan to recruit participants. The 30 healthy females who identified their interest in our study after this first public presentation were invited to a second presentation, during which individuals who had not used special skin care products, such as exfoliators, during the two weeks preceding the testing session and who agreed not to use these products over the course of the study, were enrolled. The study was conducted between October and December 2014 and was approved by the Human Research Ethics Committee, Cheng Kung University, No. 103-111-2. All participants were informed of the experimental procedure and its risks, and informed consent for participation and publication of results was obtained.

\section{Interventions}

The effects of facial threading generally last 2-3 weeks. ${ }^{16}$ Therefore, participants underwent facial threading once every 21 days to rule out the influence of the skin's normal activity. Facial threading was performed in a room with temperature $\left(24 \pm 2^{\circ} \mathrm{C}\right)$ and relative humidity $(50 \pm 5 \%)$ control. ${ }^{17}$ Before each facial threading session, participants were asked not to use any skin care cosmetics and to clean their skin with a neutral facial cleanser, with washing using distilled water after the facial threading. Each participant waited $30 \mathrm{~min}$ in the designated waiting area before and after each 30-min facial threading session to allow facial sebum secretion to normalize and stabilize.

\section{Facial threading}

Two threading practitioners, who were mother and daughter, performed all facial threading processes. Their technique was passed down from the grandmother to the mother and to the daughter, ensuring that the facial threading treatment that participants received was technically and practically similar. In addition, the two practitioners performed the facial threading using the standard operating procedure shown in figure 1, including using a fixed amount of white powder, similar threading locations, similar process durations (timed), and a similar order of procedures. Before facial threading, white powder (fine powder containing calcium carbonate obtained by grinding white marble) was applied over the participant's entire face. Thereafter, the practitioner held one end of a cotton thread with her mouth and twined the extended part of the thread around the index finger of her left hand and the four fingers of her right hand to form a triangle (Figure 2). The end held in the practitioner's mouth was used to control the pull and movement of the remaining thread by repeatedly pulling and releasing with the left and right hands, alternately.

\section{Non-invasive measurements}

Lee $^{18}$ stated that significant differences in moisture retention, melanin indices, and roughness exist in every area of the facial skin. In this study, five regions (each, $2 \mathrm{~cm} \times 2 \mathrm{~cm}$ ) were selected for testing, including the forehead, both cheeks, and both corners of the mouth (Figure 3) ${ }^{18,19}$ Each region was tested three times, and the mean value was used for analysis.

Each participant was assessed before the first facial threading and after each session of threading. First, the 3D instruments (Video Digitizer VD300 and Skin-Visioscan ${ }^{\circledR}$ VC98) were used to measure skin surface roughness. Using the built-in photomicrographic camera and data processing and analysis software, 3D graphics were obtained to determine the changes in skin texture, roughness, and vellus hair. ${ }^{20,21}$ Additionally, a multifunctional skin analyzer (Cutometer ${ }^{\circledR}$ MPA-580 PC) with a connected probe (Corneometer, CM825, 10-mm, effective depth up to $1.5 \mathrm{~mm}$ ) was used to obtain water retention indices by capacitance detection. ${ }^{22,23}$ Another connected probe was used to measure the melanin indices 


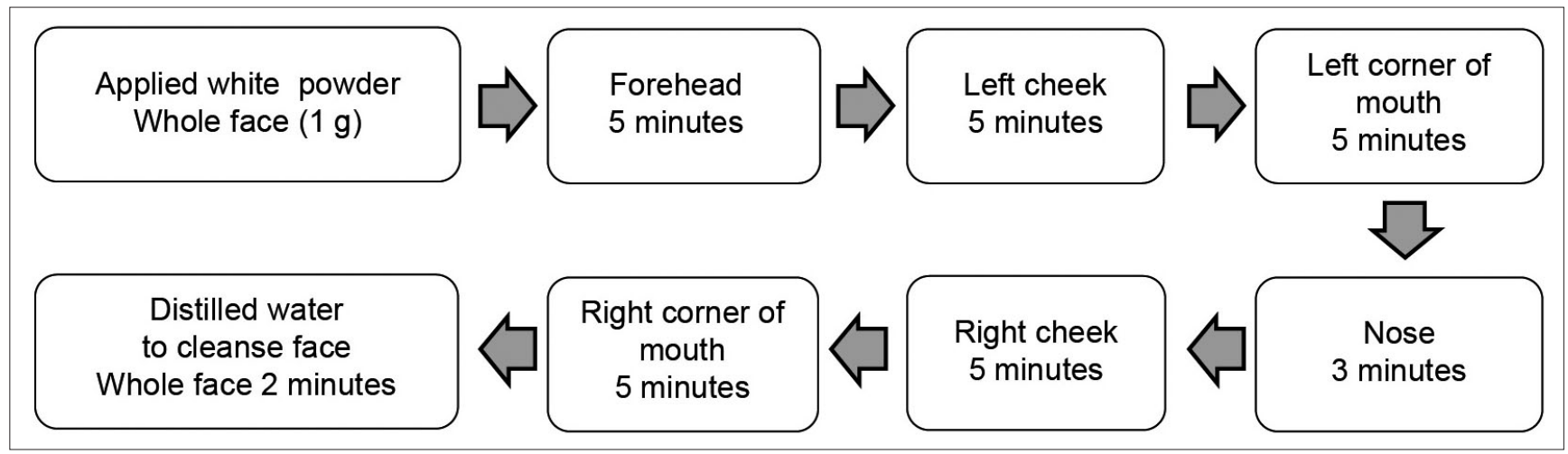

FIGURE 1: Standard facial threading procedure

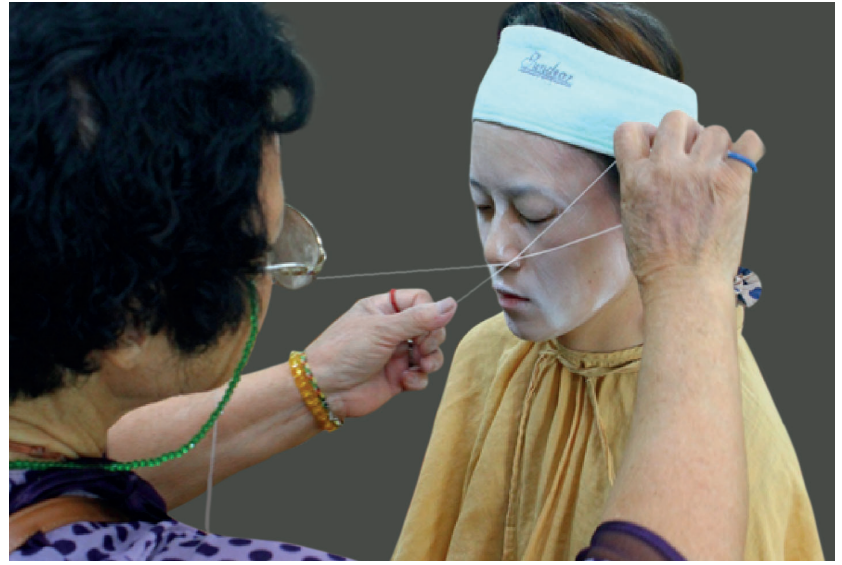

FIGURE 2: Actual illustration of facial threading

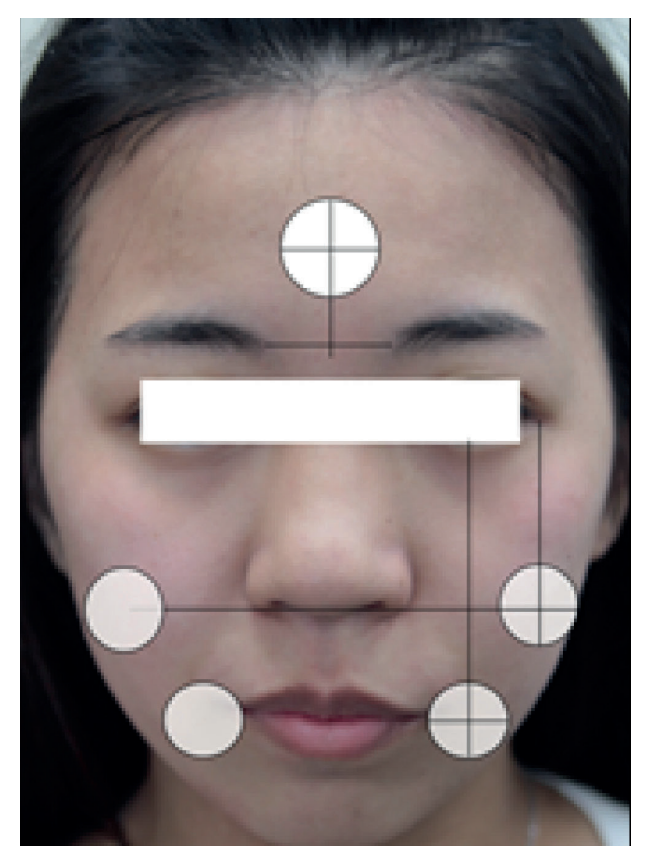

Figure 3: Locations of instrument measurement

\begin{tabular}{lccccc}
\multicolumn{7}{c}{ TABLE 1: Skin types of participants } \\
& Normal & Oily & Dry & Mixed & $\begin{array}{c}\text { Age } \\
\text { (mean } \pm \text { SD } \\
\text { years) }\end{array}$ \\
\hline $\begin{array}{l}\text { Participants } \\
\mathrm{n}=18\end{array}$ & $5.55 \%$ & $11.1 \%$ & $5.55 \%$ & $77.8 \%$ & $19.48 \pm 0.51$ \\
\hline
\end{tabular}

(narrow-band spectrophotometry; Mexameter MX18) using built-in infrared $(880 \pm 10 \mathrm{~nm})$, red $(660 \pm 3 \mathrm{~nm})$, and green $(568 \pm 3 \mathrm{~nm})$ lights to quantify skin color; the dielectric constant was expressed in arbitrary units. ${ }^{24,25}$ All research equipment was obtained from Courage + Khazaka Electronic GmbH (Cologne, Germany), and used based on operation manuals published by the manufacturer.

\section{Surveys}

Each participant completed a self-assessment questionnaire before the first facial threading session and after each subsequent session. Before facial threading, each participant selected the options that fit their skin types and then assessed their skin smoothness (very rough to very smooth on a scale of 1-5) by touch and their skin color (very dull to very light on a scale of 1-5) using a sensory perception scale.

\section{Statistical methods}

Sample size estimates were based on published statistical formulas, with a cosmetic effectiveness evaluation requiring at least 15 participants. ${ }^{17}$ Data analyses were performed using SPSS17 software (SPSS, Chicago, IL, USA). Two-tailed $t$-tests for dependent samples $(\alpha=.05)$ were used to investigate differences in skin roughness, moisture retention, and melanin indices. The Wilcoxon signed-rank test for dependent samples was used to evaluate subjectively reported differences in skin touch and color. All data are presented as means $\pm S D$, unless otherwise stated. Statistical significance was defined as p-values of $<.05$.

\section{RESULTS}

Only 20 female volunteers met the second-stage recruitment criteria. Moreover, two participants experienced allergic reactions, 
resulting in skin redness, three hours after the first facial threading and their participation was discontinued. Therefore, 18 participants completed the entire study (mean age $19.48 \pm 0.51$ years). Combination skin texture was the main type among participants (Table 1).

A decrease in skin roughness was identified at the five facial sites after facial threading, reaching statistical significance after the second threading session (Table 2). Roughness improved on the forehead and left corner of the mouth after each facial threading (Figure 4). Therefore, facial threading produced immediate and repeatable improvements in skin smoothness. In contrast, facial threading had no effect on skin moisture retention or melanin indices (Figures 5 and 6).

Improvement in subjective evaluation of the tactile feel of the skin and its color was identified after each threading session (Figure 7). The subjective evaluation of tactile feel increased from a score of $3.30 \pm 0.85$ before threading to $4.30 \pm 0.57(Z=-3.819, p<$ $.001)$ after session $1,4.22 \pm 0.64(Z=-4, p<.001)$ after session 2 , and $4.16 \pm 0.78(Z=-3.873, p<.001)$ after session 3 . The subjective score of skin color increased from $2.60 \pm 0.97$ before threading to $3.60 \pm 0.84$ $(Z=-4.025, p<.001)$ after session $1,3.78 \pm 0.73(Z=-3.827, p<.001)$ after session 2 and $3.78 \pm 0.64(Z=-3.666, p<.001)$ after session 3 .

The 3D microscope images clearly demonstrated traces of fine hair removal by the threading process, as well as apparent removal of old keratinocytes. Moreover, threading smoothed the sulci cutis and cristae cutis. After facial threading, some hairs were completely removed and some were irregularly disrupted. (Figure 8).

\section{DISCUSSION}

According to our results, participants' overall facial skin roughness improved with facial threading, which was associated with improvement in self-reported scores of skin smoothness and in quantified 3D microscope images. Although epidermal hydration is an important biophysical parameter related to the barrier function of the stratum corneum, we did not identify a significant effect of facial threading on skin hydration. ${ }^{26}$

Our measured melanin indices did not agree with the traditional view that facial threading improves skin whiteness. According to Samson et al., ${ }^{27}$ changes in skin brightness can affect the way people visually assess the youthfulness and health of skin. ${ }^{28,29}$ Facial threading does not act like skin lightening products that facilitate decreases in melanin indices and make the skin fairer. Instead, apart from removing fine facial hair, facial threading lightens skin color by removing old keratinocytes in a way similar to that of exfoliators, making the skin appear healthy and youthful.

Facial threading uses physical approaches to obtain a temporary improvement in skin smoothness and depilation. Of note, although participants with sensitive skin were excluded from our study, two participants did experience allergic reactions after the first facial threading. According to Berardesca et al., ${ }^{30,31}$ external stimuli and a thin cuticle might trigger such skin sensitivity. In addition, the unsterilized tools and materials used during facial threading (such as threads and white powder) enhance the risk of bacterial infection. ${ }^{8,9,16,32}$ Jason et al. ${ }^{5}$ recommended the following strategies to reduce the complications of facial threading, including the prevention of contagious skin diseases: proper consultation; use of sanitized equipment; use of a standardized hair removal procedure; and the selection of certified beauty parlors and cosmetologists. Regardless, facial threading does not appear appropriate for everyone,

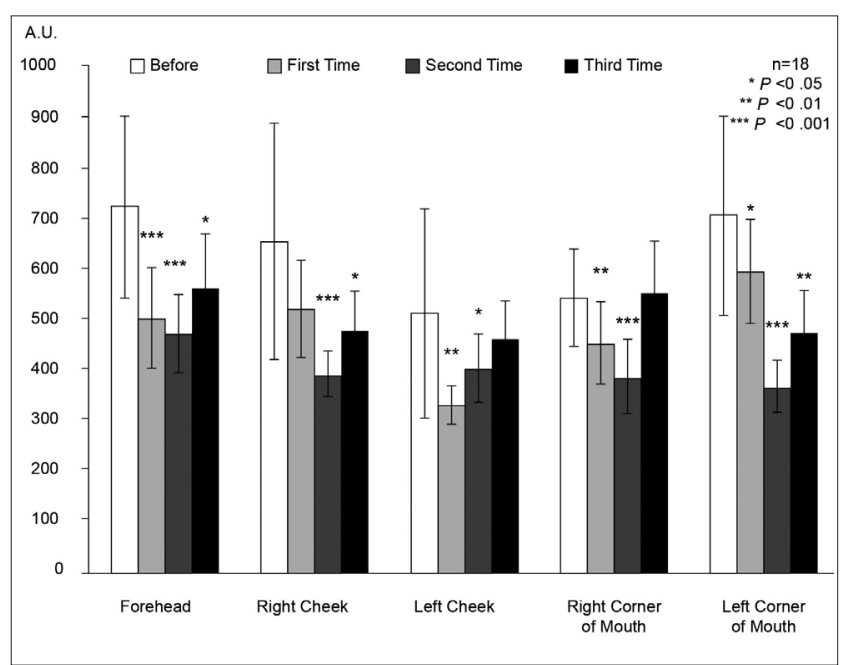

FIGURE 4: Change in skin roughness

\begin{tabular}{|c|c|c|c|c|c|c|}
\hline & & Forehead & Right cheek & Left cheek & Right mouth corner & Left mouth corner \\
\hline \multirow[t]{4}{*}{ First Threading } & Before & $717.28 \pm 179.15$ & $649.72 \pm 230.90$ & $510.44 \pm 202.89$ & $539.04 \pm 95.09$ & $701.51 \pm 195.63$ \\
\hline & After & $499.08 \pm 98.90$ & $515.83 \pm 95.57$ & $327.45 \pm 38.28$ & $448.80 \pm 82.12$ & $589.46 \pm 101.49$ \\
\hline & $\%$ & $-30.42 \%$ & $-20.61 \%$ & $-35.85 \%$ & $-16.74 \%$ & $-15.97 \%$ \\
\hline & $\mathrm{t}(17)$ & $5.918^{* * *}$ & 2.057 & $3.430 * *$ & $3.413^{* *}$ & $2.086^{*}$ \\
\hline \multirow[t]{3}{*}{ Second Threading } & After & $468.01 \pm 77.72$ & $388.19 \pm 42.78$ & $399.94 \pm 65.93$ & $383.57 \pm 71.25$ & $362.69 \pm 49.14$ \\
\hline & $\%$ & $-34.75 \%$ & $-40.25 \%$ & $-21.65 \%$ & $-28.84 \%$ & $-48.30 \%$ \\
\hline & $\mathrm{t}(17)$ & $4.918^{* * *}$ & $4.325^{* * *}$ & $2.255^{*}$ & $4.380^{* * *}$ & $7.552^{* * *}$ \\
\hline \multirow[t]{3}{*}{ Third Threading } & After & $556.48 \pm 108.34$ & $472.16 \pm 79.52$ & $458.29 \pm 73.35$ & $546.79 \pm 103.53$ & $469.87 \pm 83.88$ \\
\hline & $\%$ & $-22.42 \%$ & $-27.33 \%$ & $-10.22 \%$ & $1.44 \%$ & $-33.02 \%$ \\
\hline & $\mathrm{t}(17)$ & $2.799^{*}$ & $2.612^{*}$ & 0.976 & -0.193 & $4.357^{* * *}$ \\
\hline
\end{tabular}

Units: Arbitrary Units (A.U.); $\mathrm{n}=18 ; \mathrm{t}(17)$ : value of the t-statistic and the corresponding degree of freedom.; ${ }^{*} \mathrm{P}<.05 ; * * \mathrm{P}<.01 ; * * * \mathrm{P}<.001$ 


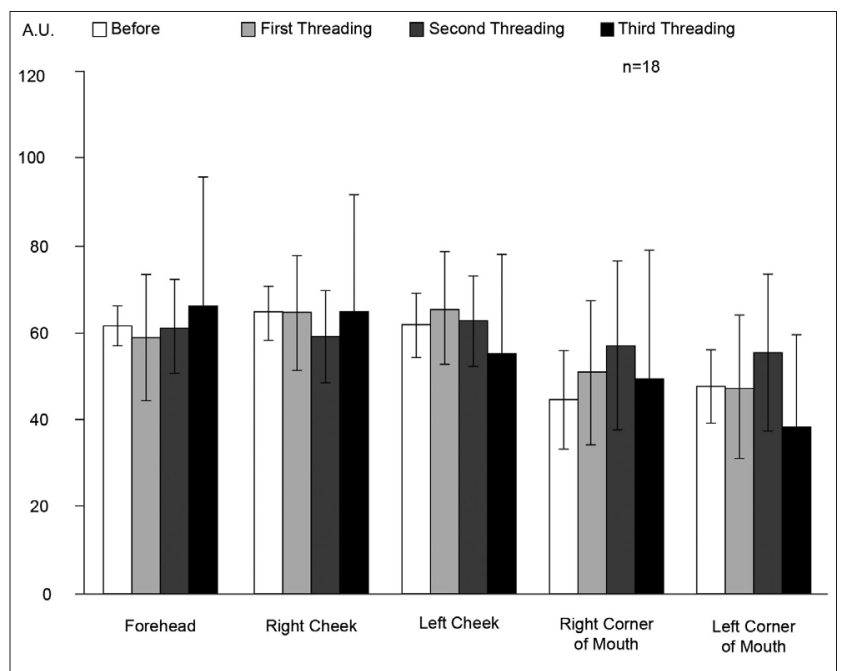

FIgure 5: Change in skin hydration

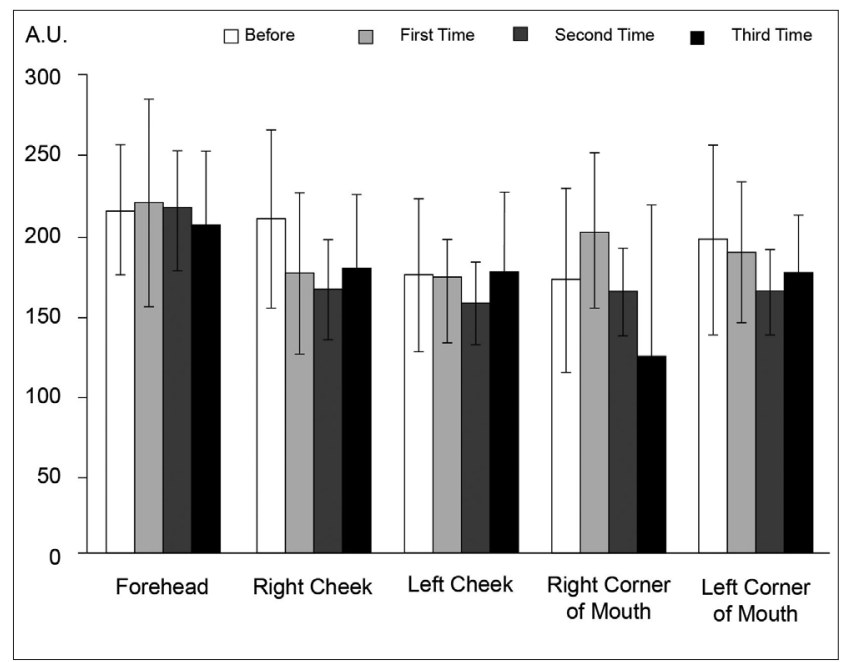

FIGURE 6: Change in melanin index of skin

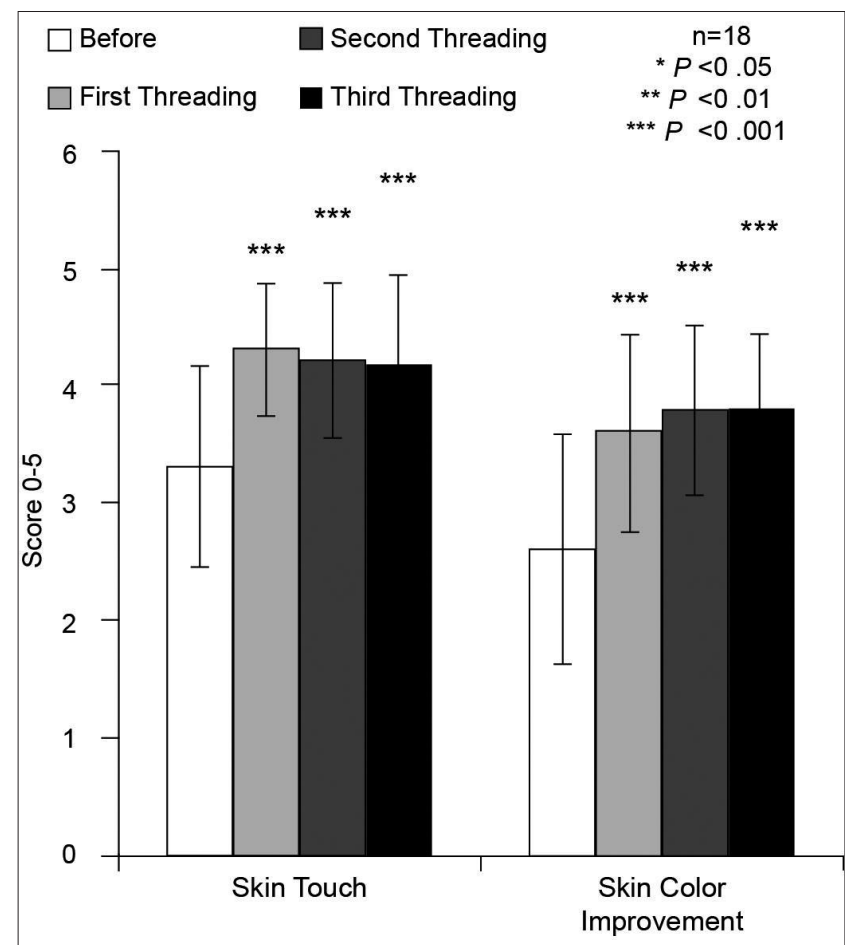

Figure 7: Change in skin tactile sensation and skin color improvement
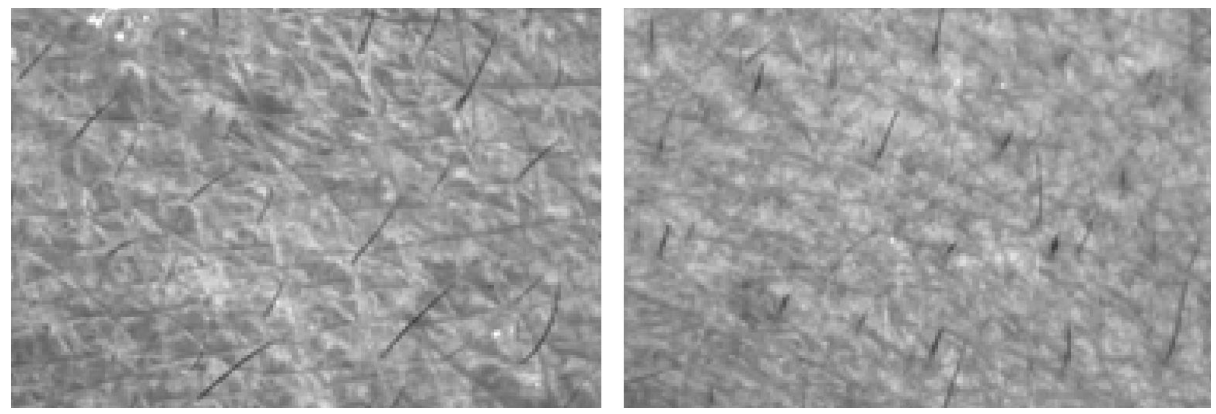

FigURE 8: 3D microscopic image after the first threading. After facial threading, some hairs were completely removed, while some were irregularly disrupted especially those with allergic and sensitive skin conditions. Thus, to avoid unwanted and unexpected side effects, the skin condition of each individual should be taken into consideration before facial threading is performed.

Although our study was limited by its design (lack of random selection), small sample size (18 participants), and absence of ethnic diversity, our results still provide some data for use by dermatologists, beauty educators, and cosmeticians.

\section{CONCLUSIONS}

Three rounds of facial threading reduced skin roughness by $26.74 \%$ and removed vellus hairs. Facial threading also improved the subjective feel of the skin and its color by one level. Future studies should include a larger and ethnically diverse cohort. Moreover, the effects of this procedure should be compared with those in a control group or with the use of other cosmetic products having similar effects. $\square$ 


\section{REFERENCES}

1. Abdel-Gawad MM, Abdel-Hamid IA, Wagner RF Jr. Khite: a non-Western technique for temporary hair removal. Int J Dermatol. 1997;36:217.

2. Hong R. Compilation of household registration laws and related terms during Japanese rule. Taipei: Aboriginal Affairs Executive Yuan; 2006. p. 137-8.

3. Lin LK. Marriage custom research in Kinmen and Fujian province. Taichung: Feng Chia University; 2012.

4. Gupta D, Thappa DM. Dermatoses due to Indian cultural practices. Indian J Dermatol. 2015:60:3-12

5. Litak J, Krunic AL, Antonijevic S, Pouryazdanparast P, Gerami P. Eyebrow epilation by threading: an increasingly popular procedure with some less-popular outcomes - a comprehensive review. Dermatol Surg. 2011;37:1051-4.

6. Lilly $E$, Kundu RV. Dermatoses secondary to Asian cultural practices. Int $J$ Dermatol. 2012;51:372-9.

7. Bloom MW, Carter EL. Bullous impetigo of the face after epilation by threading. Arch Dermatol. 2005;141:1174-5

8. Ghosh SK, Bandyopadhyay D. Molluscum contagiosum after eyebrow shaping: a beauty salon hazard. Clin Exp Dermatol. 2009;34:e339-40.

9. Sidharth S, Rahul A, Rashmi S. Cosmetic warts: pseudo-koebnerization of warts after cosmetic procedures for hair removal. J Clin Aesthet Dermatol. 2015;8:52-6.

10. Verma SB. Vitiligo koebnerized by eyebrow plucking by threading. J Cosmet Dermatol. 2002:1:214-5.

11. Firooz A, Sadr B, Babakoohi S, Sarraf-Yazdy M, Fanian F, Kazerouni-Timsar A, et al. Variation of biophysical parameters of the skin with age, gender, and body region. Scientific World Journal. 2012;2012:386936.

12. Fink B, Matts PJ. The effects of skin colour distribution and topography cues on the perception of female facial age and health. J Eur Acad Dermatol Venereol. 2008;22:493-8

13. Hong YH, Jung EY, Shin KS, Yu KW, Chang UJ, Suh HJ. Tannase-converted green tea catechins and their anti-wrinkle activity in humans. J Cosmet Dermatol. 2013;12:137-43

14. Fox LT, du Plessis J, Gerber M, van Zyl S, Boneschans B, Hamman JH. In vivo skin hydration and anti-erythema effects of Aloe vera, Aloe ferox and Aloe marlothii gel materials after single and multiple applications. Pharmacogn Mag. 2014;10(Suppl 2):S392-403.

15. Kanlayavattanakul M, Lourith $N$, Chaikul P. Jasmine rice panicle: A safe and efficient natural ingredient for skin aging treatments. J Ethnopharmacol. 2016;193:607-16.

16. Gupta S, Chaudhry M, Mahendra A, Kaur S. Eyebrow threading: a boon or a bane. Indian J Dermatol. 2011;56:715-7.

17. Zhang XM. The efficacy of cosmetic. Taipei: Five South Book Company; 2016. p. 33-5.
18. Lee MR, Nam GW, Jung YC, Park SY, Han JY, Cho JC, et al. Comparison of the skin biophysical parameters of Southeast Asia females: forehead-cheek and ethnic groups. J Eur Acad Dermatol Venereol. 2013;27:1521-6.

19. Mukherjee S, Mitra R, Maitra A, Gupta S, Kumaran S, Chakrabortty A, et al. Sebum and hydration levels in specific regions of human face significantly predict the nature and diversity of facial skin microbiome. Sci Rep. 2016;6:36062.

20. Trojahn C, Dobos G, Schario M, Ludriksone L, Blume-Peytavi U, Kottner J. Relation between skin micro-topography, roughness, and skin age. Skin Res Technol. 2015:21:69-75

21. Trojahn C, Schario M, Dobos G, Blume-Peytavi U, Kottner J. Reliability and validity of two in vivo measurements for skin surface topography in aged adults. Skin Res Technol. 2015;21:54-60.

22. Ezerskaia A, Pereira SF, Urbach HP, Verhagen R, Varghese B. Quantitative and simultaneous non-invasive measurement of skin hydration and sebum levels. Biomed Opt Express. 2016;7:2311-20.

23. Anthonissen M, Daly D, Peeters R, Van Brussel M, Fieuws S, Moortgat $P$, et al. Reliability of repeated measurements on post-burn scars with corneometer CM 825(®). Skin Res Technol. 2015;21:302-12.

24. Takiwaki H, Overgaard L, Serup J. Comparison of narrow band reflectance spectrophotometric and tristimulus colorimetric measurement of skin colour: Twenty-three anatomical sites evaluated by the Dermaspectrometer ${ }^{\circledR}$ and the Chromameter CR200. Skin Pharmacol. 1994;7:217-25.

25. Treesirichod A, Chansakulporn S, Wattanapan P. Correlation between skin color evaluation by skin color scale chart and narrowband reflectance spectrophotometer. Indian J Dermatol. 2014;59:339-42.

26. Boer M, Duchnik E, Maleszka R, Marchlewicz M. Structural and biophysical characteristics of human skin in maintaining proper epidermal barrier function. Postepy Dermatol Alergol. 2016;33:1-5

27. Samson N, Fink B, Matts PJ, Dawes NC, Weitz S. Visible changes of female facia skin surface topography in relation to age and attractiveness perception. J Cosmet Dermatol. 2010;9:79-88.

28. Matsui MS, Schalka S, Vanderover G, Fthenakis CG, Christopher J, Bombarda PC, et al. Physiological and lifestyle factors contributing to risk and severity of peri-orbital dark circles in the Brazilian population. An Bras Dermatol. 2015;90:494-503.

29. Ikino JK, Nunes DH, Silva VP, Fröde TS, Sens MM. Melasma and assessment of the quality of life in Brazilian women. An Bras Dermatol. 2015:90:196-200.

30. Berardesca E, Farage M, Maibach H. Sensitive skin: an overview. Int J Cosmet Sci. 2013;35:2-8.

31. Zaniboni MC, Samorano LP, Orfali RL, Aoki V. Skin barrier in atopic dermatitis: beyond filaggrin. An Bras Dermatol. 2016;91:472-8

32. Kumar R, Zawar V. Threading warts: a beauty parlor dermatosis. J Cosmet

Dermatol. 2007;6:279-82.

\section{AUTHORS'CONTRIBUTIONS}

Li-Ying Lin
Statistical analysis, Effective participation in research orientation, Design and planning
of the study, Preparation and writing of the manuscript, Collecting, analysis and inter-
pretation of data
Shang-Chia Chiou
Approval of the final version of the manuscript, Intellectual participation in propaedeu-
tic and/or therapeutic conduct of studied cases, Critical review of the literature, Critical
review of the manuscript

政 